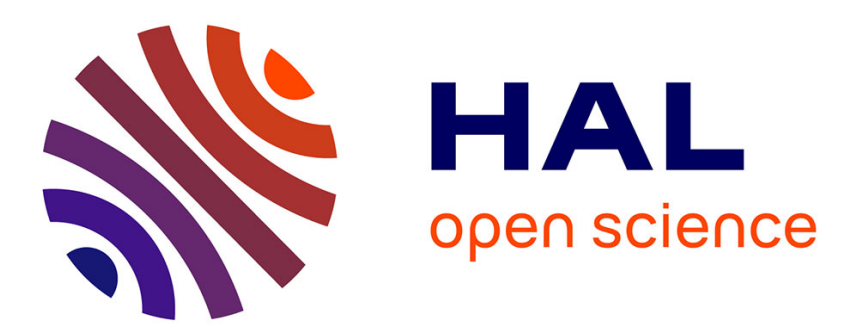

\title{
Optical detection of surface acoustic waves propagating along an interface between two solids
}

Ch. Matteï, X. Jia, G. Quentin

\section{To cite this version:}

Ch. Matteï, X. Jia, G. Quentin. Optical detection of surface acoustic waves propagating along an interface between two solids. Journal de Physique IV Proceedings, 1994, 04 (C5), pp.C5-745-C5-748. 10.1051/jp4:19945161 . jpa-00252841

\section{HAL Id: jpa-00252841 https://hal.science/jpa-00252841}

Submitted on 1 Jan 1994

HAL is a multi-disciplinary open access archive for the deposit and dissemination of scientific research documents, whether they are published or not. The documents may come from teaching and research institutions in France or abroad, or from public or private research centers.
L'archive ouverte pluridisciplinaire HAL, est destinée au dépôt et à la diffusion de documents scientifiques de niveau recherche, publiés ou non, émanant des établissements d'enseignement et de recherche français ou étrangers, des laboratoires publics ou privés. 


\title{
Optical detection of surface acoustic waves propagating along an interface between two solids
}

\author{
Ch. MATTEÏ, X. JIA and G. QUENTIN
}

Groupe de Physique des Solides, Université Paris 7, UA 17 du CNRS, Tour 23, 2 place Jussieu, 75251 Paris cedex 05, France

\begin{abstract}
Résumé: Nous proposons une méthode optique de détection des ondes d'interface fondée sur l'effet acousto-optique. En mesurant la variation d'indice optique que subit un solide transparent au passage d'une onde guidée par une interface grâce à une détection interféromètrique, il nous est possible de mesurer localement et à l'intérieur du solide le champs de déformation. Cette méthode est appliquée à l'étude de la propagation des ondes d'interface en fonction de deux conditions d'interface (glissante ou rigide) et des constantes élastiques des deux solides.
\end{abstract}

\begin{abstract}
We report an optical detection of interface waves based on the acousto-optic interaction. The variation of the index of refraction induced by a wave propagating inside a transparent media is measured using interferometric detection. This index variation is related to the compressional acoustic strain. We applied this detection method to study the propagation of interface waves under two kinds of boundary conditions (slip or rigidly bonded) between the solid substrates.
\end{abstract}

\section{INTRODUCTION}

It is well known that an acoustic wave, called Stoneley wave, can propagate without attenuation along the interface between two solids in perfect contact for some combinations of the elastic constants of the two solids. This kind of interface waves is of important interest in signal processing, nondestructive evaluation or geophysics. Most of the theoretical studies [1-3] concern the phase velocity calculation of these interface waves under different boundary contacts, e.g. "bonded" contact or "slip" contact. However, these waves are difficult to measure because of their energy confinement at the interface. Actually, most of the experiments are based on measurements of the ultrasonic reflection or transmission coefficient [4] and some others are on velocity measurements by using mode conversion methods [5].

We report here a direct measurement of the interface waves using an interferometric detection based on the acousto-optic interaction. This method, previously used to measure Rayleigh and Lamb waves [7-8], allows us to measure quantitatively pulsed acoustic strains of guided waves inside a solid, provided it is optically transparent.

\section{INTERFACE WAVES}

Stoneley [1] first studied the properties of an acoustic wave guided by the interface between two solids in perfect (rigidly bonded) contact, which implies the continuity of both compressional and shear stress at the boundary. It was shown that the amplitude of this guided wave is maximum at the interface and decays exponentially into the two solids. Many works were performed to study the existence conditions of a Stoneley wave as function of the combination of the elastic constants of the two materials. Murty [2] derived the secular equation for "slip" conditions, e.g. continuity of compressional stress and cancellation of shear stress at the interface. In a recent work, Rokhlin and Wang have put forward a model to study the interface wave at the more practical boundaries where the properties of the coupling material between the solids were taken into account. Their results are basically given for the reflection and transmission coefficients [4]. 
In the present paper, we will focused our study on the two extreme cases: rigidly bonded and slip conditions. For each boundary conditions, the calculation leads to a secular equation which admits different types of solutions for the phase velocity depending on the elastic constant combination. When a real value of the velocity exists, a Stoneley mode propagates, that is to say, a wave localised at the interface without attenuation in the propagation direction. In the case of the bonded conditions, Pilant [3] investigated a large range of combination of elastic constant. When no real value of the velocity exists, depending on the combination of elastic constant, two kinds of waves can propagate: an attenuated interface wave (localised at the interface with attenuation along the propagation) or a leaky Rayleigh wave (radiation of a Rayleigh wave propagating in a substrate into the other substrate). To our knowledge this kind of investigation doesn't exist for the other boundary conditions, however the existence of similar waves may be expected for the slip boundary conditions.

Now let us consider only the Stoneley mode. Murty [2] showed how the conditions of existence is modified for different boundary conditions. Figure 1 shows the results of the computation for two Poisson solids $(\lambda 1=\mu 1, \lambda 2=\mu 2)$ as function of the elastic constants of the materials. It appears that the two different boundary conditions give different ranges of existence : the range is far larger for the slip conditions.

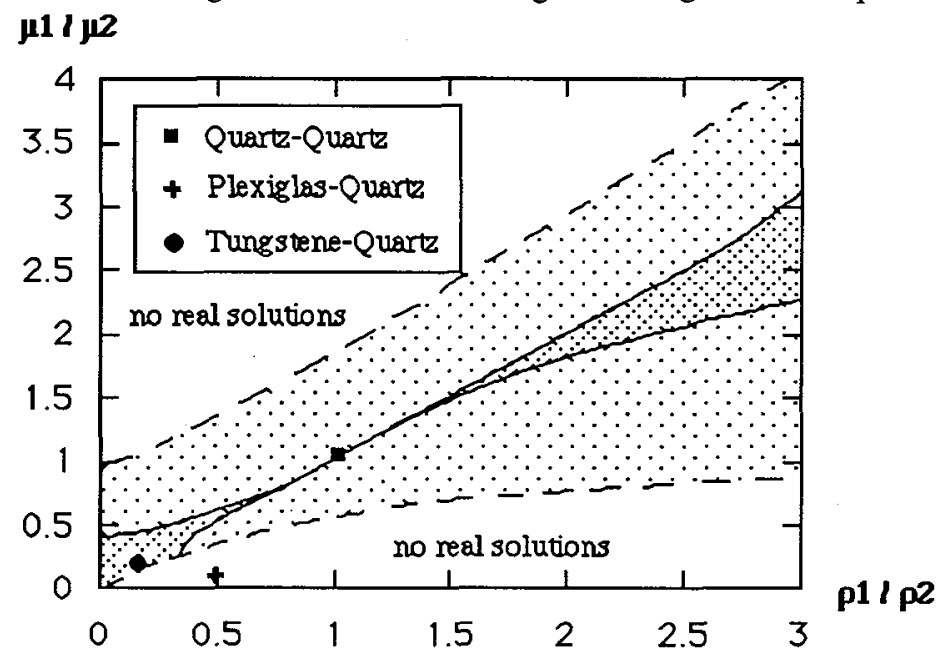

figure 1. The range of existence of interface waves for Poisson half-spaces. In the range limited by the two dashed lines, interface waves exist for slip conditions. In the range limited by the solid line, interface waves exist for bonded conditions (from Murty [2]). The points correspond to the pair of materials used in our experiments .

\section{INTERFEROMETRIC MEASUREMENTS OF INTERFACE WAVES STRAIN}

To measure these interface waves, we used an optical interferometric method based on the acoustooptic interaction which has been previously applied to Rayleigh and Lamb waves measurements $[7,8]$. The principle of this method is to measure the variation of the index of refraction due to the acoustic strain inside a transparent solid when a guided acoustic wave propagates. For a guided wave with the displacements components in the sagittal plan, the associated strain tensor $S i j$ has three non zero components. The variation of the dielectric permittivity tensor induced by the strains, due to the photoelastic effect, is:

$$
\Delta \varepsilon_{\mathrm{ij}}=-\left(\varepsilon_{0}\right)^{2} \mathrm{p}_{\mathrm{ijkl}} \mathrm{S}_{\mathrm{kl}}
$$

where $\varepsilon_{0}$ is the permittivity constant of the solid, $\Delta \varepsilon_{i j}$ is the variation due to the strain Skl and pijkl is the photoelastic coefficient. If a laser beam cross normally an acoustic wave, the variation of strain will induce an optical phase shift which can be expressed [6]:

$$
\Delta \Phi=\left(\frac{\omega}{\mathrm{c}}\right) \Delta \overline{\mathrm{n}} \mathrm{L}=\left(\frac{\omega}{\mathrm{c}}\right) \mathrm{L} \frac{\mathrm{n}^{3}}{2}\left(\mathrm{p}_{11}+\mathrm{p}_{12}\right)\left(\mathrm{S}_{\mathrm{xx}}+\mathrm{S}_{\mathrm{zz}}\right)
$$

where $\omega, c$ are respectively the angular frequency and the speed of the light, $L$ is the width of the acoustic beam, $\Delta \mathrm{n}$ is the variation of the index of refraction (related to the dielectric permittivity tensor) proportional to the compressional acoustic strain. By using an heterodyne interferometer [6], we can obtain this optical phase shift and consequently the compressional strain inside the solid. In fused quartz a detection sensitivity of the order of $1 \mathrm{mV} / 10-7$ ( $1 \mathrm{mV}$ corresponding to a 10-7 strain) is obtained. More details about this optical interferometric method are available in Ref[6]. 


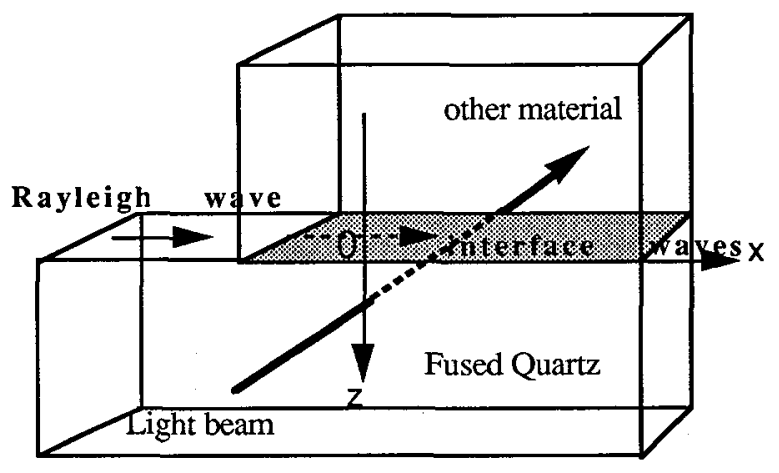

Figure 2: Lay-out of the optical detection of an interface wave.
Figure 2 shows schematically the lay-out of our experiment. The interface wave is generated by the incidence of a Rayleigh wave via the mode conversion. This latter is generated by a wedge transducer [5].The lower transparent solid is fused quartz, the upper solid can be a transparent or opaque solid. Three combinations are used in the experiment : Fused quartzFused quartz, Fused quartz-Plexiglas, Fused quartz-Tungsten. Figure 1 shows the positions of these combinations. Only the last combination admits interface waves for both boundary conditions. Slip conditions were realised using a very thin layer of water between the optically polished materials. The bonded conditions were achieved by gluing the two substrates. Broad band pulse or tone-burst electrical excitation were used. The laser beam can scan in the $\mathrm{x}$ - or $\mathrm{z}$-direction in such a way that it is possible to make velocity or attenuation measurement in the propagation direction as well as penetration measurements of the interface waves in the z-direction.

\section{EXPERIMENTAL RESULTS AND DISCUSSION}

One of the difficulties in the interface wave detection by the classical methods is to verify its interface nature. The present optical method allows us to measure locally the field distribution of the wave strain and to determine if has a field confinement close to the interface. Figure 3 shows the strain wave form of transient pulses propagating at the slip interface between two fused quartz substrates measured at different depths, on each side of the interface. The amplitude of the wave decays with distance from the interface and tends to zero around three millimetres from the interface for a tone-burst of $1 \mathrm{MHz}$ central frequency. This shows that the wave energy is confined at the interface. Moreover, a $\pi$-phase shift is observed, in fig 3, between the strain signals measured at each side of the solids. This is due to the flexural characteristics of the boundary motion generated by the interface waves at the both sides. This phase shift is observed on figure 3 comparing the two curves on the left, with a very good accuracy. The propagation velocity measured is $3400 \mathrm{~m} / \mathrm{s}$, in good agreement with the theory.

When bonded conditions are present at the interface, the interface wave tends to disappear, the velocity measured was $3610 \mathrm{~m} / \mathrm{s}$, close to the shear velocity in fused quartz $(3600 \mathrm{~m} / \mathrm{s})$. The wave becomes more bulk-like and penetrates further inside the solids. This corresponds to Murty's calculation for the existence conditions.

For the Tungsten-Fused quartz combination, an interface wave propagates for both bonded and slip conditions (Figures 4 and 5). The decay in fused quartz was observed and velocities of $2730 \mathrm{~m} / \mathrm{s}$ for slip conditions and $2860 \mathrm{~m} / \mathrm{s}$ for bonded conditions were respectively measured, which are in agreement with the calculated velocities. This material combination is particularly interesting because the velocity of the interface wave is quite lower than the Rayleigh wave velocity of the two substrates.

The experiment done with the fused quartz-plexiglas combination showed that, for both boundary conditions no interface wave propagates. The initial Rayleigh wave is attenuated by radiation into the plexiglas as predicted by Murty and propagates with a velocity of $3300 \mathrm{~m} / \mathrm{s}$ which corresponds to the Rayleigh wave velocity in fused quartz.

In summary, the strain field of acoustic waves propagating along the interface between to solids have been measured by optical interferometric detection. The experimental results obtained for slip and bonded conditions are in good agreement with the theory. This shows that the present interferometric method provides a novel means for studying interface wave, especially for the boundary conditions where the viscoelastic coupling layer is taken into account. 

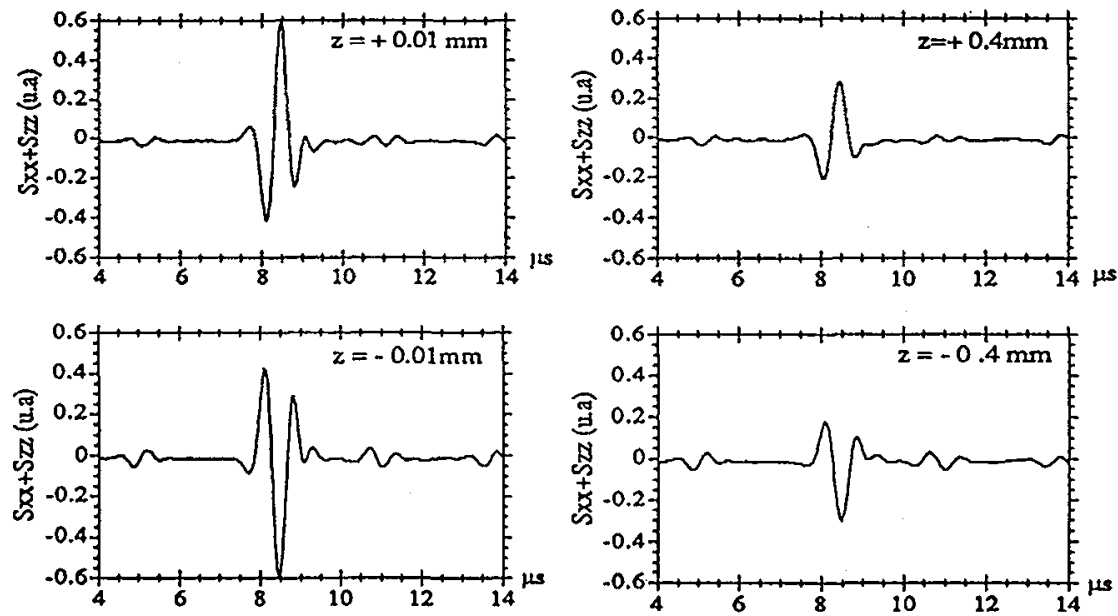

Figure 3 . Strain waveform of the interface wave propagating at the slip interface between two quartz half-spaces measured at different depths from the interface. The two upper curves shows the signal in the upper medium, the two lower curves shows the signal in the lower medium.
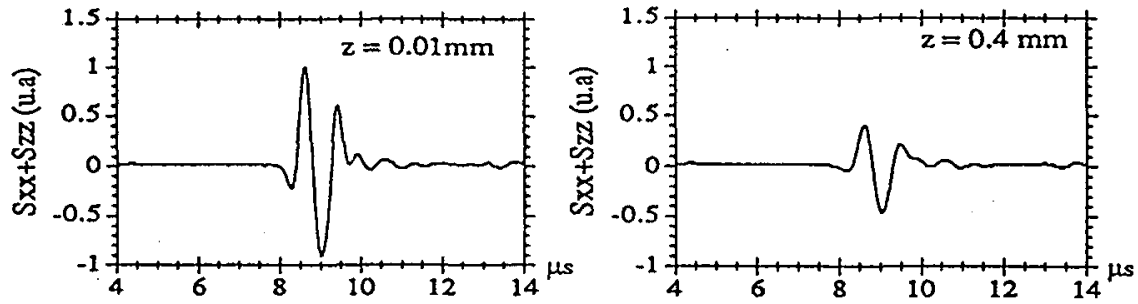

Figure 4.Strain waveform of the interface wave propagating at the slip interface between quartz and Tungsten half-spaces measured at different depths from the interface.
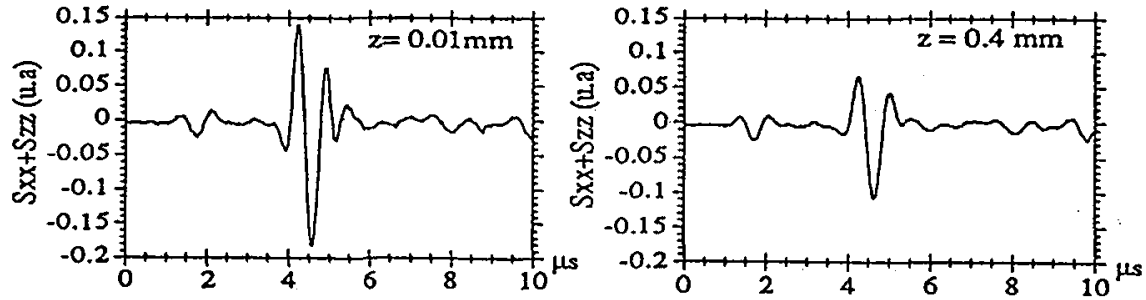

Figure 5. Strain waveform of the interface wave propagating at the rigidly bonded interface between quartz and Tungsten half-spaces measured at different depths from the interface.

\section{References:}

[1] Stoneley R., Proc. Roy. Soc. London (1924), A-106, 416.

[2] Murty G.S., Physics of the Earth and Planetary Interiors, 11(1975), 65-79.

[3] Pilant W., Bull. of Seism. Soc. of Am. 62 (1), (1972), 285-299.

[4] S.I Rokhlin and Y.J Wang, J. Acoust. Soc. Am 89 (2),(1991), 503-515.

[5] P.W Staecker and W.C Wang, J. Acoust. Soc. Am 53 (1),(1973), 65-74.

[6] D.Royer and E Dieulesaint,"Optical detection of sub-angström transient mechanical displacements", Proceedings of 1986 Ultrasonics Symposium, New York,1986.

[7] Mattei Ch, Jia X. and Quentin G., " Quantitative strain measurements of surface acoustic waves by optical interferometry",Review of Progress in Quantitative Nondestructive Evaluation., D.O Thomson Ed., Brunswick Maine USA, 1993 (Plenum Press).

[8] Jia X.,Mattei Ch and Quentin G.,"Optical interferometric measurement of guided surface acoustic waves strains inside a solid", Proceedings of 1993 Ultrasonics Symposium, Baltimore USA, 1993. 